\title{
BMJ Open How digital health solutions align with the roles and functions that support hospital to home transitions for older adults: a rapid review study protocol
}

\author{
Hardeep Singh (D) ," Alana Armas, ${ }^{1,2}$ Susan Law, ${ }^{2,3}$ Terence Tang (D) ," \\ Carolyn Steele Gray, ${ }^{1,2}$ Heather V Cunningham, ${ }^{4}$ Rachel Thombs, ${ }^{1,2}$ Moriah Ellen, ${ }^{2,5}$ \\ Jasvinei Sritharan, ${ }^{1}$ Jason X Nie ${ }^{3}$ Donna Plett, ${ }^{3}$ Carlotta Micaela Jarach, ${ }^{6}$ \\ Kednapa Thavorn, ${ }^{7,8}$ Michelle L A Nelson ${ }^{1,2}$
}

To cite: Singh H, Armas A, Law S, et al. How digital health solutions align with the roles and functions that support hospital to home transitions for older adults: a rapid review study protocol. BMJ Open 2021;11:e045596. doi:10.1136/ bmjopen-2020-045596

- Prepublication history for this paper is available online. To view these files, please visit the journal online (http://dx.doi. org/10.1136/bmjopen-2020045596).

Received 06 0ctober 2020 Revised 09 February 2021 Accepted 10 February 2021
Check for updates

(C) Author(s) (or their employer(s)) 2021. Re-use permitted under CC BY-NC. No commercial re-use. See rights and permissions. Published by BMJ.

For numbered affiliations see end of article.

Correspondence to Dr Michelle L A Nelson; michelle.nelson@sinaihealth.ca

\section{ABSTRACT}

Introduction Older adults may experience challenges during the hospital to home transitions that could be mitigated by digital health solutions. However, to promote adoption in practice and realise benefits, there is a need to specify how digital health solutions contribute to hospital to home transitions, particularly pertinent in this era of social distancing. This rapid review will: (1) elucidate the various roles and functions that have been developed to support hospital to home transitions of care, (2) identify existing digital health solutions that support hospital to home transitions of care, (3) identify gaps and new opportunities where digital health solutions can support these roles and functions and (4) create recommendations that will inform the design and structure of future digital health interventions that support hospital to home transitions for older adults (eg, the pre-trial results of the Digital Bridge intervention; ClinicalTrials.gov Identifier: NCT04287192).

Methods and analysis A two-phase rapid review will be conducted to meet identified aims. In phase 1, a selective literature review will be used to generate a conceptual map of the roles and functions of individuals that support hospital to home transitions for older adults. In phase 2, a search on MEDLINE, EMBASE and CINAHL will identify literature on digital health solutions that support hospital to home transitions. The ways in which digital health solutions can support the roles and functions that facilitate these transitions will then be mapped in the analysis and generation of findings.

Ethics and dissemination This protocol is a review of the literature and does not involve human subjects, and therefore, does not require ethics approval. This review will permit the identification of gaps and new opportunities for digital processes and platforms that enable care transitions and can help inform the design and implementation of future digital health interventions. Review findings will be disseminated through publications and presentations to key stakeholders.

\section{INTRODUCTION}

The transition from hospital to home can be a vulnerable, stressful and challenging period
Strengths and limitations of this study

- A rapid review methodology will allow us to generate timely results to inform the design of digital health interventions.

- Limitations of the rapid review methodology are important to consider, including streamlined processes and the potential to miss relevant information.

- A large research team that includes content experts can minimise bias associated with a rapid review methodology.

- Terminology, such as digital health and navigators, is inconsistently used in literature, which can lead to missed information.

for older adults. ${ }^{1-10}$ Within 30 days of hospital discharge, nearly one-fifth of older adults are rehospitalised with an acute medical problem that may be unrelated to their previous hospitalisation, resulting in prevalent and costly rehospitalisations. ${ }^{4} 7$ During transitions of care, many patients have depleted physiological reserves, which can affect their ability to effectively mitigate health threats. ${ }^{4}$ Other aggravating factors, such as a medication errors or poor reconciliation, reduced ability to manage their health due to sleep deprivation, nutritional issues, pain and other discomfort, a lack of information and resources or information overload, can contribute to a patient's rehospitalisation during this period. ${ }^{145}$ In addition, older adults may have complex care needs and underlying or new functional and/or cognitive impairments that can impact their adjustment/adaption to daily life after transitioning home from the hospital. ${ }^{2} 10-12$ These various multifactorial and cumulative stressors ${ }^{12}$ leave patients vulnerable after a hospitalisation (ie, "posthospital syndrome'). ${ }^{49}$ A meta-summary 
by Hestevik et al found that older adults had negative experiences during transitions; they described their transition experience as 'insecure', 'unsafe', as well as even 'dangerous'. ${ }^{10}$ An ageing global population ${ }^{13}$ means that more and more older adults will undergo this transition from hospital to home, making efforts to improve these transitions critically important. ${ }^{1415}$

A transition of care is defined as 'a set of actions designed to ensure the coordination and continuity of healthcare as patients transfer between different locations or different levels of care within the same location'. ${ }^{16}{ }^{17}$ More specifically, the transition of care from the hospital to 'independent community living' (or the home) involves activities that span across organisational boundaries and involves multiple interprofessional groups. ${ }^{18}$ Transitions home from the hospital must be individualised to each patient based on their needs and situation; for some patients, these transitions are not a linear trajectory, but rather a stressful circuitous journey that includes premature starts (eg, patients discharged from hospital prior to being ready for discharge), delays (eg, equipment and community services not setup on time or information not provided in a timely manner during a handoff) and repeated steps (eg, repeated patient assessments). ${ }^{18}$ As transitions of care are a 'problematic junction in a patient's care management,' seamless transitions are a high priority goal in healthcare. ${ }^{19}$

Well-coordinated and planned hospital to home transitions lower healthcare costs, hospital readmission rates, incidents or risk of injury (eg, falls) and medication errors. ${ }^{315} 17$ 20-23 On the other hand, poorly coordinated hospital to home transitions-caused by breakdowns in processes and ineffective handoff of information between healthcare providers-can lead to: poor patient experiences, miscommunication among healthcare providers about treatment plans, fragmented care, duplicative testing, medication errors and missed appointment follow-ups with physicians. ${ }^{23}$ Additionally, poorly coordinated hospital to home transitions can increase healthcare spending and negative patient outcomes. ${ }^{724-26}$ In fact, up to half of the adverse events that occur during hospital to home transitions are preventable, or the severity of adverse events could have been decreased by taking proactive corrective action. ${ }^{27}$ These actions may include providing patients with adequate education about their medical condition and treatment, and implementing efforts to effectively communicate specific information to the patient and community physician (eg, posthospital follow-up and red flags they should watch for). ${ }^{25-27}$

A nurse-led transitional care model that extends care from the hospital to community has demonstrated reductions in rehospitalisations and healthcare costs. ${ }^{28}$ The intervention involves a comprehensive, multidisciplinary intervention that begins in-hospital and continues in the community ${ }^{28}{ }^{29}$ Appropriate, timely and person-centred communication between hospital and community settings is a pivotal aspect of an effective transition process. ${ }^{28}$ 30-33 For instance, patients require health-related information (eg, new medication regimens, diagnostic tests, procedures, medication changes during hospitalisation) and follow-up instructions to primary care physicians to anticipate/reduce the frequency and severity of adverse events, as well as information/resources to manage their social problems that could lead to poor patient outcomes. ${ }^{23283435}$

Given the complexity of the healthcare system, multiple key individuals are needed to produce seamless handoffs and transitions of care.$^{36}$ A review by Manderson $e t$ $a l$ identified 13 different system navigator titles held by healthcare professionals that aimed to assist older adults during the transitions of care. The functions of these system navigators were heterogenous and the authors identified a need for further clarification of these roles. ${ }^{37}$ A related review by Dohan and Schrag found the roles of patient navigators differed from other roles (eg, case managers and advocates) that support transitions of care, but found patient navigators were poorly defined in the literature..$^{38}$ Abrashkin et al identified the critical roles of physicians, nurses, pharmacists, social workers, case managers and patient navigators in producing seamless transitions of care. ${ }^{36}$ To ensure seamless care transitions, a key function during care transitions is producing wellcoordinated handoffs (ie, passing important patientrelated information from one provider to another). ${ }^{39} 40$ Handoffs can be between providers in the same organisation (eg, hospital physician to hospital nurse) and/or between providers in different settings (eg, hospital physician to community physician); however, handoffs tend to be marked with challenges/failure. ${ }^{39} 40$ Various key functions must be carried out by providers during care transitions to produce well-coordinated care transitions. ${ }^{24}$ Given the heterogeneity in the roles and functions that support transitions of care, a conceptual map of specific roles, their functions and how these roles interconnect or work together to support transitions is needed.

For the purposes of this protocol, we have defined 'roles' as the job titles held by individuals (navigator, nurse, etc), and 'functions' as the duties/responsibilities performed by the individual during older adults' transition home from the hospital (eg, produce a discharge summary, provide caregiver education, etc).$^{41}$

Digital health technologies have been proposed as a mechanism to facilitate seamless handoffs ${ }^{42-44}$ and to support patients during this vulnerable transition period. ${ }^{45}{ }^{46}$ Digital health is defined by Iyawa $e t$ al as 'the use of information and communication technologies to monitor and improve the well-being and health of patients and to empower patients in the management of their health and that of their families. ${ }^{, 47}$ These technologies have numerous capabilities, including the potential to facilitate effective communication needed as part of high-quality transition support ${ }^{48}$ and enable monitoring of patients' health status in the community. ${ }^{31}{ }^{49}$ Moreover, these technologies can support existing roles and functions aimed to facilitate transitions of care, such as patient navigators. ${ }^{32} 4850$ During the COVID-19 pandemic, there has been rapid uptake of digital health technologies in 
healthcare and it is anticipated that this trend will persist beyond the COVID-19 pandemic. ${ }^{51}$

However, a challenge is that many current digital health solutions are not meeting the clinical needs of healthcare providers and care needs of patients. ${ }^{39-58}$ For instance, digital health technologies have limited success for reasons such as user frustrations ${ }^{56}$ or a loss of interest. ${ }^{55}$ Another possibility for the limited success of these technologies is they do not fit (or fulfil) the users' needs in the context that the technology was intended for (eg, do not fit with existing clinical workflow). ${ }^{39} 5758$ There is an inseparable relationship between digital health solutions and the context or landscape in which they operate. ${ }^{59}$ As such, successful adoption of health technology is dependent on how well care providers see the technology complementing with their current role. ${ }^{6061}$ Thus, a strong understanding of the roles and function of individuals supporting hospital to home transitions can inform our thinking about how digital health solutions can support the needs of the various individuals involved in these transitions. Implementing digital health solutions to coordinate care across healthcare organisations is a complex task that requires many stakeholders to work together in order to address the particular processes and policies between organisations, while also considering the needs of patients. ${ }^{62}$

To meet user need, the development process for digital health solutions should incorporate evidence of effectiveness and the engagement of users in the design process. ${ }^{6163}$ Research has shown that health technology fails to be adopted if the needs of end-users are not considered in the design and implementation of health technology ${ }^{64}$ Unfortunately, software tends to be designed for younger individuals and may not meet the needs of older adults, who can have lower digital literacy, reduced motor control, age-related cognitive changes and/or agerelated sensory changes. ${ }^{65}$ Future digital health interventions may benefit from greater efforts directed towards improving acceptance and widespread adoption of digital health solutions by intended technology users (eg, older adults and clinicians).

The Digital Bridge (ClinicalTrials.gov ID: NCT04287192) ${ }^{46}$ is an upcoming digital health intervention that our research team is working on, which aims to improve communication across clinical settings. Digital Bridge will be designed with input from multiple intended users (ie, patients, caregivers, hospital clinicians and primary care clinicians) ${ }^{46}$ as well as the evidence generated from this review.

To enable our team to respond to the challenges with creating digital health solutions identified above, the overarching research questions that this review seeks to answer are: how have digital health solutions supported the various roles and functions of individuals involved in hospital to home care transitions, and where are there gaps and opportunities? The current review will map the various roles and functions that have been developed to support hospital to home transitions of care and then contrast how various digital health solutions have been used in these contexts to support transitions from hospital to home. Moreover, we will identify gaps and new opportunities for digital health solutions and platforms to support and facilitate current roles and functions involved in these transitions using a structured rapid review; these gaps and opportunities will be addressed in the design of the Digital Bridge. ${ }^{46}$ A structured rapid review is appropriate to produce timely results ${ }^{66}$ that will inform how digital technologies can effectively support transitions while informing future development work, as well as areas where the design can be improved. Therefore, the specific objectives of this rapid review include the following: (1) to elucidate the various roles and functions that have been developed to support hospital to home transition of care, (2) to identify existing digital health solutions that support hospital to home transitions of care, (3) to identify gaps and new opportunities where digital health solutions can support these roles and functions and (4) to create recommendations that will inform the design and structure of future digital health interventions that support hospital to home transitions of care for older adults, such as the Digital Bridge intervention. ${ }^{46}$

\section{METHODS}

This two-phase rapid review ${ }^{67}$ will combine a selective literature review (as described below) of the roles and functions that support hospital to home transitions of care, with a structured rapid review of the digital health solutions that support these roles and functions involved in hospital to home transitions of care. The research questions for each phase are specified below.

\section{Phase 1}

Research questions

In phase 1, the research questions guiding our pursuit of foundational knowledge, which will build foundational knowledge for phase 2, include the following:

- What are the roles and functions of individuals that facilitate hospital to home transitions of care for older adults?

- What process and outcome measures have been used to assess the effectiveness of specific roles that facilitate hospital to home transitions of care for older adults?

\section{Literature search}

A selective literature search will be conducted by research team members-this approach limits a review of the literature to 'key studies that make a significant contribution to our understanding' of a particular topic. ${ }^{68}{ }^{69} \mathrm{~A}$ search strategy will be developed for popular multidisciplinary databases, Medline and Google Scholar, to identify any type of study that defines roles and functions related to hospital to home transition (eg, acute care to home, rehabilitation to home) for older adult populations ( $\geq 65$ years of age). A selective literature review ${ }^{70}$ is appropriate 
here as our intent is to assess the collective evidence and build foundational knowledge on the topic, ${ }^{72}$ which will be expanded on in phase 2. Concepts relating to navigation, hospital to home transition of care and older adults will be searched. As there is a lack of standardised terminology and definitions for healthcare professional and non-healthcare professional navigators, the search will consist of search terms identified by previous literature, including 'navigator' or 'patient navigator' or 'system navigator' or 'care facilitator' or 'transitional care' or 'postdischarge support' or 'care coordinator' or 'service coordination' or 'volunteer navigator' or 'peer navigator' or 'discharge coordinator' or 'informal care' or 'caregiver'. ${ }^{37} 73-76$ In addition to this, content experts will be consulted to ensure key studies (including grey literature) have been included (ie, desk drawer search). Hand searches will be conducted through the references of key studies that will be retrieved ${ }^{6777}$ This knowledge is essential to identifying opportunities and gaps for digital health solutions that will be identified in phase 2 .

\section{Data extraction}

Reviewers will chart data from all included studies using a data charting form, which will be developed collectively by the research team. This form will be pilot tested on a random selection of included studies. Data extracted from each included study will relate to the study type, study purpose, name and definition of the roles, details of their function, the setting/context they operate in and the process and outcome measures used to measure their effectiveness. The data extraction form will be refined iteratively throughout the data charting process to ensure all relevant data will be extracted. ${ }^{78}$

\section{Summarising and reporting}

A thematic analysis ${ }^{79}$ will be used to derive key findings/ themes. The thematic analysis will involve two researchers independently generating codes related to the type, function and definition of roles and functions described in each study. Through discussions, the researchers will consolidate their codes to create a final set of codes. Following coding, the two researchers will work together to group codes based on patterns and relationships between the codes to form themes. ${ }^{79}$ The results of the studies will be organised into themes, as well as presented in a tabular form as per data extraction. This is an appropriate analysis approach as the results are expected to include a range of different study types and designs. ${ }^{80} \mathrm{~A}$ conceptual map ${ }^{81}$ providing a visual representation of the themes (ie, roles and functions) supporting hospital to home transitions will be developed. Concept maps are 'two-dimensional diagrams showing hierarchical relationships between concepts of a body of knowledge and they derive their existence from the conceptual structure of this body. ${ }^{82} 83$ Concept maps have several advantages, including: (1) the ability to define a central idea (ie, roles/ functions) by positioning it in the centre of the map; (2) the ability to provide information on the relative position of each idea (eg, whether the role or function is in the hospital or community); (3) a visual depiction of relationships between the key ideas (ie, between the roles/ functions) to make it easier to understand the complex relationships between concepts and (4) the ability to present all information, including complex ideas, on a single page, making it easier to interpret and ensuring that recall and review are more efficient. ${ }^{83}$ Conceptual maps are appropriate outputs for our aim in phase 1 , as they can be used to examine different aspects of the roles within health systems/services in depth, and to compare and contrast theoretical frameworks and approaches, and facilitate analysis across the system, particularly in cases where 'data and ideas are captured using different taxonomies. ${ }^{84}$ The map will be used to guide data extraction and knowledge synthesis in phase $2 .^{85}$ We anticipate that we will generate two related concept maps: (1) describing the roles and (2) describing the function. The relationship between these two maps will also be depicted. Information on the outcome measures used to determine the effectiveness of the roles will be descriptively reported.

\section{Phase 2}

Phase 2 will consist of a rapid review ${ }^{67}$ of literature pertaining to digital health solutions that facilitate hospital to home transition of care for older adults.

\section{Research questions}

In phase 2, the following questions will be addressed:

- What is known about the characteristics and attributes of digital health solutions for older adults that support hospital to home transitions of care?

- What are the relevant facilitators and barriers to adoption of digital health solutions to facilitate hospital to home transitions for older adults?

- What process and outcome measures are used to determine the effectiveness of digital health interventions for older adults, and how do they compare to outcome measures used to determine the effectiveness of the roles and functions developed to support hospital to home transitions of care for older adults?

\section{Literature search}

A search of electronic databases will be conducted to examine digital health solutions that support the transition of care from hospital to home. This search will include terms related to the concepts of digital health, navigation and transition of care from hospital to home. These search terms will be partly informed by previous reviews. ${ }^{1786}$ The search will be limited to high income countries because the purpose of this review is to inform future digital health interventions, such as the Digital Bridge intervention, ${ }^{46}$ which will be applied in a high income country. ${ }^{87}$ We will also limit the search to older adult populations as we anticipate that digital health technologies not tested with older adults may not meet their needs. An information specialist (HVC) developed and ran the search first on Medline (see online supplemental 
material for the phase 2 medline search). To ensure the search is rigorous, this Medline strategy was peer-reviewed using the Peer Review of Electronic Search Strategies ${ }^{88}$ by a second Information Specialist who was not involved in this project and validated using a set of relevant studies. A search on MEDLINE on 26 November 2020 generated 10, 304 citations. This search will be translated for EMBASE and CINAHL. These databases are widely used for searches on health-related topics, and were deemed the most relevant databases for the scope of this search by the research team. ${ }^{89} 90$ Additional search strategies will include: (1) scanning the reference lists of included studies, (2) consulting experts in the field to identify relevant studies ${ }^{77}$ and (3) searching conference proceedings published within the last 2 years of key digital health and e-health focused conferences (in Canada, USA and Europe) to identify digital health solutions that have been developed but not yet published.

\section{Screening and study selection}

The results from all databases will be imported into Endnote. Following this, any duplicates will be removed, and the remaining studies will be imported into Covidence; a software platform that allows screening and data extraction from studies. The process to identify eligible studies will begin with title and abstract screening, followed by a full-text review. $^{78}$

Title and abstract screening: Titles and abstracts of the search results will be scanned to identify studies that meet the inclusion and exclusion criteria outlined in table 1 .

The inclusion and exclusion criteria will be pilot tested by three content experts and the reviewers and, if needed, refined after increased familiarity with the data. ${ }^{781}$ The inter-rater reliability (IRR) will be determined based on a random sample of search results that will be independently reviewed by the reviewers. Given the expertise and experience of the research team a kappa statistic of $\geq 0.80$, indicating almost perfect agreement, will be acceptable $^{92}$ for reviewers to independently screen. ${ }^{93}$ If the IRR is below the threshold, the criteria will be refined again until the minimum IRR threshold has been reached. ${ }^{781}$ Post hoc development of detailed inclusion and exclusion criteria is consistent with traditional scoping review methodology; wherein the criteria are developed in an iterative manner. ${ }^{78}$ Once final inclusion and exclusion criteria has been established, reviewers will then independently apply the criteria when screening the titles and abstracts of the remaining studies. The screeners will indicate which studies to include, exclude or ones that are uncertain. Any disagreements will be resolved through discussions with the team. As recommended by Levac et $a l$, the reviewers will meet at the beginning, midpoint and final stages of the abstract review process to ensure there is substantial agreement on which studies to include. ${ }^{78}$

Full-text screening: After the title and abstract screening, single reviewers will conduct full-text reviews of the potentially eligible studies. ${ }^{78}$

\section{Data extraction}

A data extraction form will be created collectively by the research team based on the template for intervention description and replication (TIDierR) reporting checklist. ${ }^{94}$ The TIDieR checklist is suitable for extracting data relating to the research aims: describing the intervention details and characteristics (eg, TIDieR items 1-10), implementation factors (as per the TIDieR defined components of implementation) (eg, TIDieR items 3, 6, 7-11) and outcome measures (eg, TIDieR items 11-12). ${ }^{94}$ The reviewers will first test the data extraction form on a randomly selected sample of studies. The form may be further refined during the data extraction process and any refinements needed will be discussed with the research team. ${ }^{78}$ Interventions that measure the effectiveness of various roles that support transitions of care often use a variety of different outcome measures. ${ }^{37}$ Thus, we are interested in capturing all outcomes reported in phase 2, so that we will be able to compare them to the outcomes used to measure successfully facilitated transitions of care identified in phase 1 . We will assess the quality of studies using the Mixed Methods Appraisal Tool (MMAT). ${ }^{95} 96$ The MMAT is appropriate for this review as it is a critical appraisal tool that can be used to concurrently assess and compare quality across different study designs (quantitative, qualitative and mixed-methods studies).$^{96-99}$ The MMAT has demonstrated moderate to excellent reliability ${ }^{97}$ and has been effectively used in prior reviews that include studies with multiple study designs. ${ }^{99-102}$ For scoring, the authors advise detailing the ratings of each criterion rather than calculating a total score. ${ }^{96}$

\section{Summarising and reporting}

Knowledge synthesis will consist of analysing the data, reporting results and applying meaning to the results. ${ }^{78}$ We will begin with a textual description of the digital health solutions. Following this, we will qualitatively analyse the heterogeneity across and within these studies, such as differences in the features of the digital health solutions, methodology, study design, sample, study setting, dimensions of context and outcomes. ${ }^{79} 103$ We will explore relationships within and between studies using concept maps. ${ }^{78}$ We will consider how the quality of the included studies impacts the recommendations that we make for the design of future digital health interventions.

Next, we will integrate the results from phase 1, which will allow us to relate the application of digital health to existing roles and functions involved in transitions of care. We will expand the conceptual maps from phase 1 by annotating the functions and attributes of digital health solutions found in phase 2 and visually interpret how these can support the roles and functions. We will also visually identify gaps in existing digital health solutions and opportunities for improvement. However, consistent with other reviews, we anticipate that the knowledge synthesis will be an iterative process and dependent on the literature found. ${ }^{78} 104$ 
Table 1 Phase 2 inclusion and exclusion criteria

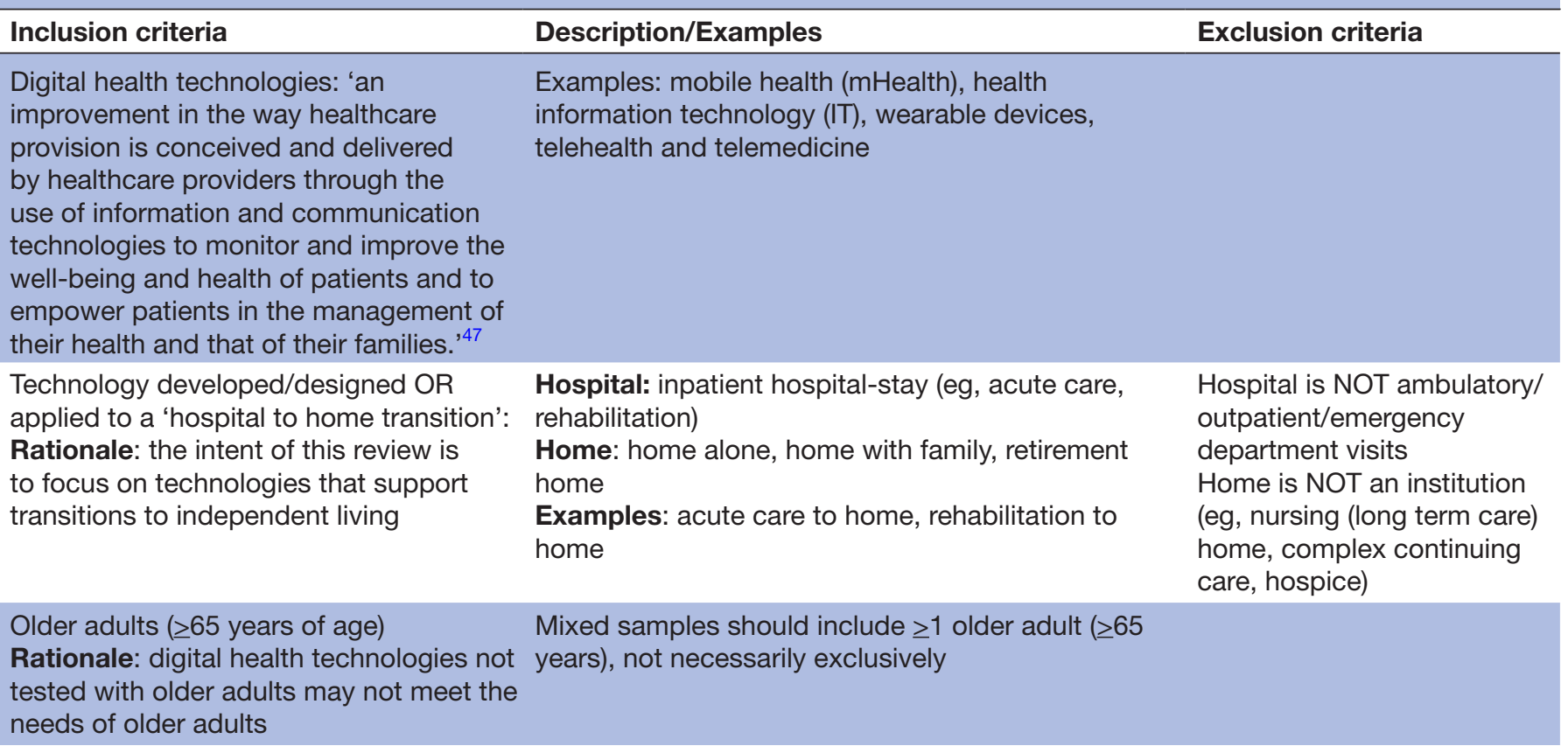

Available in English

Rationale: time and resource

constraints $^{91}$

\section{Published $\geq$ year 2010}

Rationale: older technologies may be obsolete/outdated

Empirical study: Studies that answer a research question by 'obtaining direct, observable information from the world, rather than, for example, by theorising, or by reasoning, or by arguing from first principles. The key concept is 'observable information about (some aspect of) the world'. ${ }^{108}$

Rationale: we are interested in information pertaining to outcomes and processes; non-empirical studies are not data-driven and tend to focus on theories/methods. ${ }^{109-111}$ As results will be used to support future digital health interventions, the included citations must provide insights into outcomes and/or implementation considerations within practice. Theoretical papers may not reflect these insights.

\section{Examples of empirical designs: Pilot Studies} (eg, feasibility or Utility studies), Action Research, Case Studies, Ethnography, Evaluation Methods, Evaluation, Research Experiments, Focus Groups, Field Studies, Interviews, Mail Surveys, Mixed Methods Research, Naturalistic Observation, Online Surveys, Participant Observation, Participatory Research, Qualitative Research, Questionnaires Research, Statistical Analysis, Statistical Studies, Telephone Surveys
Studies are not data driven and tend to focus on theories/methods and reviews collate data from multiple studies making it difficult to extract details from individual studies such as opinion letters, commentaries, editorials, protocols

Reviews (literature, systematic or scoping review) 
Table 1 Continued

\begin{tabular}{|c|c|}
\hline Inclusion criteria & Description/Examples \\
\hline $\begin{array}{l}\text { Applied in a high-income country } \\
\text { Rationale: our intent is to inform future } \\
\text { digital health interventions, such as the } \\
\text { Digital Bridge intervention, }{ }^{46} \text { which will } \\
\text { be applied in developed or 'high-income' } \\
\text { country; contextual, health system factors } \\
\text { and technologies will differ in lower } \\
\text { income countries. }\end{array}$ & $\begin{array}{l}\text { Andorra, Antigua and Barbuda, Aruba, Australia, } \\
\text { Austria, The Bahamas, Bahrain, Barbados, Belgium, } \\
\text { Bermuda, British Virgin Islands, Brunei Darussalam, } \\
\text { Canada, Cayman Islands, Channel Islands, Chile, } \\
\text { Croatia, Curacao, Cypress, Czech Republic, } \\
\text { Denmark, Estonia, Faroe Islands, Finland, France, } \\
\text { French Polynesia, Germany, Gibraltar, Greece, } \\
\text { Greenland, Guam, Hong Kong, Hungary, Iceland, } \\
\text { Ireland, Isle of Man, Israel, Italy, Japan, Korea } \\
\text { Rep., Kuwait, Latvia, Liechtenstein, Lithuania, } \\
\text { Luxembourg, Macao Sar, China, Malta, Mauritius, } \\
\text { Monaco, Nauru, Netherlands, New Caledonia, New } \\
\text { Zealand, Northern Mariana Islands, Norway, Oman, } \\
\text { Palau, Panama, Poland, Portugal, Puerto Rico, Qatar, } \\
\text { Romania, San Marino, Saudi Arabia, Seychelles, } \\
\text { Singapore, Sint Maarten, Slovak Republic, Slovenia, } \\
\text { Spain, St. Kitts and Nevis, St. Martin, Sweden, } \\
\text { Switzerland, Taiwan China, Trinidad and Tobago, } \\
\text { Turks and Caicos Islands, United Arab Emirates, } \\
\text { United Kingdom, United States, Uruguay, Virgin } \\
\text { Islands (USA) }\end{array}$ \\
\hline
\end{tabular}

\section{Report production and dissemination}

The Preferred Reporting Items for Systematic Reviews and Meta-Analyses Protocols checklist will be used as a reporting template to increase the transparency of this review. ${ }^{105}$ Preliminary research findings will be shared with content experts and Digital Bridge's Knowledge Translation Advisory Committee for feedback on how to meaningfully analyse and present the data findings. The results of this review will be shared with healthcare professionals and researchers in the form of publications and presentations. In addition, the results will be shared with the Digital Health Enabling Integrated Care special interest group, which is part of the International Foundation for Integrated Care. Members of this group include an international community of healthcare professionals and researchers.

Based on the results of phase 1 and phase 2, a set of key recommendations will be created to guide the design and implementation of digital health tools intended to support care transitions from hospital to home. These recommendations will first be applied to the Digital Bridge intervention. ${ }^{46}$ These recommendations will be shared with the Digital Bridge research team and patient advisory committees of the two multisite healthcare institutions which are planned sites for the Digital Bridge intervention. A report that highlights key recommendations will be generated to guide the design of the Digital Bridge intervention. ${ }^{46}$ The report will include the conceptual maps generated in phases 1 and 2, which will provide the foundation for the codesign process of this intervention, as well as highlight considerations for the technology design/functionality and implementation factors. This report will be publicly available for the design of future digital health interventions.
Patient and public involvement

Patients or the public were not involved in the design, conduct, reporting or dissemination of our research.

\section{DISCUSSION}

Given the rapid uptake of digital health interventions amidst the COVID-19 pandemic, ${ }^{106}$ this review will be timely and relevant to the rapidly evolving current healthcare context. The strengths of this review lie in its potential to guide improvements to current and future digital health interventions. In particular, this review will synthesise evidence that can guide the design of digital health interventions, improve the understanding of the role of technology in the context of the care transition roles and functions, and identify potential gaps for current and future digital health innovation. Another noteworthy strength of this review is that a concrete knowledge translation plan exists to support the application of the review's results into the codesign of an upcoming digital health intervention.

Nonetheless, potential limitations related to methodological choices in this review are important to consider. First, phase 1 is a selective literature review to build foundational knowledge about key concepts and contextual factors; it is not intended to be a comprehensive review of the literature. As such, the review may not capture all relevant studies. However, we are confident that it will build a sufficient conceptual map of the roles and functions of individuals involved in hospital to home transitions of care to guide our work in phase 2. Second, our search strategy (eg, imposing language and date restrictions) can potentially exclude relevant studies. However, given the purpose of this review is to provide a summary 
of the evidence to inform the design of the Digital Bridge intervention, ${ }^{46}$ such restrictions were necessary given the available time and resources to complete this preliminary step, and as are typical of rapid reviews. We anticipate that despite the limitations, we will learn what is needed to inform our planned design process. Third, due to the scope of this review we will limit the search to older adult populations. Although older adults may have differing health technology needs, ${ }^{6465}$ it is possible that we could miss digital health interventions that are developed and applied in younger populations but still applicable to older adult populations. Future research could compare digital health interventions developed and applied in older populations to younger populations. Fourth, rapid reviews are susceptible to bias as a result of streamlining processes of systematic reviews ${ }^{91}$; however, having a large research team, that includes technical expertise from a medical librarian and content experts, will minimise bias associated with streamlining processes in this review. Finally, digital health is a term inconsistently used in existing literature ${ }^{107}$ and as a result, we may potentially miss relevant studies. To address this, we have adopted a broad definition of digital health in our search strategy to ensure we minimise missed studies and made the definition and search strategy transparent in this review.

\section{Author affiliations}

${ }^{1}$ Bridgepoint Collaboratory for Research and Innovation, Lunenfeld-Tanenbaum Research Institute, Sinai Health System, Toronto, Ontario, Canada

${ }^{2}$ Institute of Health Policy, Management \& Evaluation, Dalla Lana School of Public Health, University of Toronto, Toronto, Ontario, Canada

${ }^{3}$ Institute for Better Health, Trillium Health Partners, Mississauga, Ontario, Canada ${ }^{4}$ Gerstein Science Information Centre, University of Toronto, Toronto, Ontario, Canada

${ }^{5}$ Department of Health Systems Management, Guilford Glazer Faculty of Business and Management and Faculty of Health Sciences, Ben-Gurion University of the Negev, Beer-Sheva, Israel

${ }^{6}$ Health Systems Management, Ben-Gurion University of the Negev Faculty of Health Sciences, Beer Sheva, Israel

${ }^{7}$ Clinical Epidemiology Program, Ottawa Hospital Research Institute, Ottawa,

Ontario, Canada

${ }^{8}$ School of Epidemiology and Public Health, University of Ottawa, Ottawa, Ontario, Canada

\section{Twitter Carlotta Micaela Jarach @CarlottaJarach}

Contributors HS: data curation, methodology, writing-original draft; AA: data curation, methodology, writing-original draft, reviewing and editing; SL: conceptualisation, methodology, writing—review and editing; TT: conceptualisation, methodology, writing — review and editing, supervision; CSG: conceptualisation, methodology, writing —review and editing, supervision; HVC: data curation, investigation, writing - review and editing; RT: data curation, writing — review and editing; ME: methodology, writing — review and editing; JS: writing—review and editing; JXN: visualisation, writing—review and editing; DP: writing — review and editing; CMJ: writing — review and editing; KT: writing — review and editing; MLAN: conceptualisation, methodology, supervision, writingreview and editing.

Funding This work was supported by a Canadian Institutes of Health Research (CIHR) Fellowship to HS and a Team Grant: Transitions in Care from the Canadian Institutes of Health Research.

Competing interests None declared.

Patient consent for publication Not required.

Provenance and peer review Not commissioned; externally peer reviewed.
Supplemental material This content has been supplied by the author(s). It has not been vetted by BMJ Publishing Group Limited (BMJ) and may not have been peer-reviewed. Any opinions or recommendations discussed are solely those of the author(s) and are not endorsed by BMJ. BMJ disclaims all liability and responsibility arising from any reliance placed on the content. Where the content includes any translated material, BMJ does not warrant the accuracy and reliability of the translations (including but not limited to local regulations, clinical guidelines, terminology, drug names and drug dosages), and is not responsible for any error and/or omissions arising from translation and adaptation or otherwise.

Open access This is an open access article distributed in accordance with the Creative Commons Attribution Non Commercial (CC BY-NC 4.0) license, which permits others to distribute, remix, adapt, build upon this work non-commercially, and license their derivative works on different terms, provided the original work is properly cited, appropriate credit is given, any changes made indicated, and the use is non-commercial. See: http://creativecommons.org/licenses/by-nc/4.0/.

ORCID iDs

Hardeep Singh http://orcid.org/0000-0002-7429-5580

Terence Tang http://orcid.org/0000-0002-1735-7298

\section{REFERENCES}

1 Werner NE, Tong M, Borkenhagen A, et al. Performance-shaping factors affecting older adults' hospital-to-home transition success: a systems approach. Gerontologist 2019;59:303-14.

2 Neiterman E, Wodchis W, Bourgeault I. Experiences of older adults in transition from hospital to community. Can J Aging 2014;34:1-10.

3 Naylor MD. Transitional care for older adults: a cost-effective model. LDI Issue Brief 2004:9:1-4.

4 Krumholz HM. Post-Hospital syndrome - an acquired, transient condition of generalized risk. N Engl J Med 2013;368:100-2.

5 Foust JB, Naylor MD, Bixby MB, et al. Medication problems occurring at hospital discharge among older adults with heart failure. Res Gerontol Nurs 2012;5:25-33.

6 Andreasen J, Lund $\mathrm{H}$, Aadahl M, et al. The experience of daily life of acutely admitted frail elderly patients one week after discharge from the hospital. Int J Qual Stud Health Well-being 2015;10:27370.

7 Jencks SF, Williams MV, Coleman EA. Rehospitalizations among patients in the Medicare fee-for-service program. $N$ Engl J Med 2009;360:1418-28.

8 Mesquita ET, Cruz LN, Mariano BM, et al. Post-Hospital syndrome: a new challenge in cardiovascular practice. Arq Bras Cardiol 2015:105:540-4.

9 van Seben R, Reichardt LA, Essink DR, et al. "I Feel Worn Out, as if I Neglected Myself": Older Patients' Perspectives on Posthospital Symptoms After Acute Hospitalization. Gerontologist 2019;59:315-26.

10 Hestevik $\mathrm{CH}$, Molin M, Debesay J, et al. Older persons' experiences of adapting to daily life at home after hospital discharge: a qualitative metasummary. BMC Health Serv Res 2019;19:224.

11 Dilworth-Anderson P, Hilliard TS, Williams S, et al. A Contextual Conceptualization on Transitions of Care for Older Persons: $<>$ Shaping the Direction of Care</I\&gt. annu rev gerontol geriatr 2011;31:1-14.

12 King BD. Functional decline in hospitalized elders. Medsurg Nurs 2006;15:265-71. quiz 72

13 United Nations. World Population Ageing 2017 - Highlights New York: UN, 2017. Available: https://www.un.org/en/development/ desa/population/publications/pdf/ageing/WPA2017_Highlights.pdf [Accessed July 8 2020].

14 Gustafsson M, Kristensson J, Holst G, et al. Case managers for older persons with multi-morbidity and their everyday work - a focused ethnography. BMC Health Serv Res 2013;13:496.

15 Naylor MD, Aiken LH, Kurtzman ET, et al. The importance of transitional care in achieving health reform. Health Aff 2011;30:746-54.

16 Coleman EA, Boult C. Improving the quality of transitional care for persons with complex care needs. J Am Geriatr Soc 2003;51:556-7.

17 Kalu ME, Maximos M, Sengiad S, et al. The role of rehabilitation professionals in care transitions for older adults: a scoping review. Phys Occup Ther Geriatr 2019;37:123-50.

18 Waring J, Marshall F, Bishop S. Health Services and Delivery Research. An ethnographic study of knowledge sharing across the boundaries between care processes, services and organisations: the contributions to 'safe' hospital discharge. Southampton (UK: NIHR Journals Library, 2014. 
19 Enderlin CA, McLeskey N, Rooker JL, et al. Review of current conceptual models and frameworks to guide transitions of care in older adults. Geriatr Nurs 2013;34:47-52.

20 Brand CA, Jones CT, Lowe AJ, et al. A transitional care service for elderly chronic disease patients at risk of readmission. Aust. Health Review 2004;28:275-84.

21 Verhaegh KJ, MacNeil-Vroomen JL, Eslami S, et al. Transitional care interventions prevent Hospital readmissions for adults with chronic illnesses. Health Aff 2014;33:1531-9.

22 Albert NM. A systematic review of transitional-care strategies to reduce rehospitalization in patients with heart failure. Heart Lung 2016:45:100-13.

23 Mansukhani RP, Bridgeman MB, Candelario D. Exploring transitional care: evidence-based strategies for improving provider communication and reducing readmissions. PT 2015;40:690-4.

24 Naylor MD, Brooten D, Campbell R. Comprehensive discharge planning and home follow-up of hospitalized elders: a randomized clinical trial. JAMA 1999;281:613-20.

25 Forster AJ, Murff HJ, Peterson JF, et al. The incidence and severity of adverse events affecting patients after discharge from the hospital. Ann Intern Med 2003;138:161-7.

26 Coleman EA, Smith JD, Frank JC, et al. Preparing patients and caregivers to participate in care delivered across settings: the care transitions intervention. J Am Geriatr Soc 2004;52:1817-25.

27 Forster AJ, Clark HD, Menard A. Adverse events among medical patients after discharge from hospital. Can Med Assoc $J$ 2004;170:345.

28 Naylor MD, Brooten DA, Campbell RL, et al. Transitional care of older adults hospitalized with heart failure: a randomized, controlled trial. J Am Geriatr Soc 2004;52:675-84.

29 Hirschman KB, Shaid E, McCauley K, et al. Continuity of care: the transitional care model. Online J Issues Nurs 2015;20:1.

30 Gaikwad R, Warren J. The role of home-based information and communications technology interventions in chronic disease management: a systematic literature review. Health Informatics $J$ 2009:15:122-46.

31 Steele Gray C, Gill A, Khan Al, et al. The electronic patient reported outcome tool: testing usability and feasibility of a mobile APP and portal to support care for patients with complex chronic disease and disability in primary care settings. JMIR Mhealth Uhealth 2016;4:e58.

32 Haque S, Ebron S, Bailey B, et al. Use of health information technology among patient Navigators in community health interventions. Perspect Health Inf Manag 2019;16:1a.

33 Patel B, Johnston M, Cookson N, et al. Interprofessional communication of clinicians using a mobile phone APP: a randomized crossover trial using simulated patients. J Med Internet Res 2016;18:e79.

34 Kripalani S, LeFevre F, Phillips CO. Deficits in communication and information transfer between hospital-based and primary care physicians: implications for patient safety and continuity of care. JAMA 2007;297:831-41.

35 Wong JD, Bajcar JM, Wong GG, et al. Medication reconciliation at hospital discharge: evaluating discrepancies. Ann Pharmacother 2008;42:1373-9.

36 Abrashkin KA, Cho HJ, Torgalkar S, et al. Improving transitions of care from hospital to home: what works? Mt Sinai J Med 2012;79:535-44.

37 Manderson B, Mcmurray J, Piraino E, et al. Navigation roles support chronically ill older adults through healthcare transitions: a systematic review of the literature. Health Soc Care Community 2012;20:113-27.

38 Dohan D, Schrag D. Using navigators to improve care of underserved patients: current practices and approaches. Cancer 2005; 104:848-55.

39 Abraham J, Kannampallil T, Patel B. Ensuring patient safety in care transitions: an empirical evaluation of a handoff intervention tool. AMIA Annu Symp Proc 2012;2012:17-26.

40 Cohen MD, Hilligoss PB. The published literature on handoffs in hospitals: deficiencies identified in an extensive review. BMJ Qual Saf 2010;19:493-7.

41 Kelly KJ, Doucet S, Luke A. Exploring the roles, functions, and background of patient navigators and case managers: a scoping review. Int J Nurs Stud 2019;98:27-47.

42 Van Eaton EG, Horvath KD, Lober WB, et al. A randomized, controlled trial evaluating the impact of a computerized rounding and sign-out system on continuity of care and resident work hours. J Am Coll Surg 2005;200:538-45.

43 Stein DM, Wrenn JO, Johnson SB. Signout: a collaborative document with implications for the future of clinical information systems. AMIA Annu Symp Proc 2007;2007:696-700.
44 Flanagan ME, Patterson ES, Frankel RM, et al. Evaluation of a physician informatics tool to improve patient handoffs. Journal of the American Medical Informatics Association 2009;16:509-15.

45 Frail CK, Garza OW, Haas AL. Experience with technologysupported transitions of care to improve medication use. Journal of the American Pharmacists Association 2016;56:568-72.

46 Steele Gray C, Tang T, Armas A, et al. Building a digital bridge to support patient-centered care transitions from hospital to home for older adults with complex care needs: protocol for a CoDesign, implementation, and evaluation study. JMIR Res Protoc 2020;9:e20220.

47 lyawa GE, Herselman M, Botha A. Digital health innovation ecosystems: from systematic literature review to conceptual framework. Procedia Comput Sci 2016;100:244-52.

48 McAlearney AS, Sieck CJ, Hefner JL, et al. High touch and high tech (HT2) proposal: transforming patient engagement throughout the continuum of care by engaging patients with portal technology at the bedside. JMIR Res Protoc 2016;5:e221.

49 Martín-Lesende I, Orruño E, Mateos M, et al. Telemonitoring inhome complex chronic patients from primary care in routine clinical practice: impact on healthcare resources use. European Journal of General Practice 2017;23:136-43.

50 Darkins A, Ryan P, Kobb R, et al. Care Coordination/Home telehealth: the systematic implementation of health informatics, home telehealth, and disease management to support the care of veteran patients with chronic conditions. Telemedicine and e-Health 2008; 14:1118-26.

51 Peek N, Sujan M, Scott P. Digital health and care in pandemic times: impact of COVID-19. BMJ Health Care Inform 2020;27:e100166.

52 Zhou L, Bao J, Watzlaf V, et al. Barriers to and facilitators of the use of mobile health Apps from a security perspective: mixed-methods study. JMIR Mhealth Uhealth 2019;7:e11223.

53 Sharma A, Harrington RA, McClellan MB, et al. Using Digital Health Technology to Better Generate Evidence and Deliver EvidenceBased Care. J Am Coll Cardiol 2018;71:2680-90.

54 Marcolino MS, Oliveira JAQ, D'Agostino M, et al. The impact of mHealth interventions: systematic review of systematic reviews. JMIR Mhealth Uhealth 2018;6:e23.

55 Krebs P, Duncan DT. Health APP use among US mobile phone owners: a national survey. JMIR Mhealth Uhealth 2015;3:e101.

56 Sarkar U, Gourley GI, Lyles CR, et al. Usability of commercially available mobile applications for diverse patients. J Gen Intern Med 2016;31:1417-26.

57 Tsiknakis M, Kouroubali A. Organizational factors affecting successful adoption of innovative eHealth services: a case study employing the FITT framework. Int J Med Inform 2009;78:39-52.

58 Hans PK, Gray CS, Gill A, et al. The provider perspective: investigating the effect of the electronic patient-reported outcome (ePRO) mobile application and portal on primary care provider workflow. Prim Health Care Res Dev 2018;19:151-64.

59 Meskó B, Drobni Z, Bényei Éva, et al. Digital health is a cultural transformation of traditional healthcare. Mhealth 2017;3:38.

60 Sharma U, Reed J, Doyle C. Challenges in evaluating telehealth through RCT- the problem of randomization. Stud Health Technol Inform 2012;180:323-7.

61 Shaw J, Agarwal P, Desveaux L, et al. Beyond "implementation": digital health innovation and service design. npj Digital Med 2018;1:48.

62 Winthereik BR, Bansler JP. Connecting practices: ICT infrastructures to support integrated care. Int J Integr Care 2007;7:e16.

63 Mathews SC, McShea MJ, Hanley CL, et al. Digital health: a path to validation. npj Digit Med 2019;2:38

64 Birnbaum F, Lewis D, Rosen RK, et al. Patient engagement and the design of digital health. Acad Emerg Med 2015;22:754-6.

65 Stojmenova E, Imperl B, Ž T. User-Centred e-health: engaging users into the e-health design process 2012. BLED 2012;38.

66 Khangura S, Konnyu K, Cushman R, et al. Evidence summaries: the evolution of a rapid review approach. Syst Rev 2012;1:10.

67 Tricco AL, EV; Straus SE. Rapid reviews to strengthen health policy and systems: a practical guide. Geneva: World Health Organization, Alliance for Health Policy and Systems Research, 2017.

68 Bryant SL. The information needs and information seeking behaviour of family doctors: a selective literature review. Health $\mathrm{Libr}$ Rev 2000;17:83-90.

69 Linda NS, Phetlhu DR, Klopper HC. Significance of literature when constructing a theory: a selective literature review. Afr J Phys Health Educ Recreat Dance 2014:502-12.

70 Shyman E. Please wait, processing: a selective literature review of the neurological understanding of emotional processing in ASD and its potential contribution to Neuroeducation. Brain Sci 2017;7:153. 
71 Colombo AF, Lee P, Karney BW. A selective literature review of transient-based leak detection methods. J Hydro-environ Res 2009;2:212-27.

72 Snyder $\mathrm{H}$. Literature review as a research methodology: an overview and guidelines. J Bus Res 2019;104:333-9.

73 Lim SY, Jarvenpaa SL, Lanham HJ. Barriers to Interorganizational knowledge transfer in Post-Hospital care transitions: review and directions for information systems research. J Manag Inf Syst 2015;32:48-74.

74 Prieto-Centurion V, Basu S, Bracken N, et al. Design of the patient navigator to reduce readmissions (partner) study: a pragmatic clinical effectiveness trial. Contemp Clin Trials Commun 2019;15:100420.

75 Geerlings SW, MARGRIET POT A, TWISK JOSWR, et al. Predicting transitions in the use of informal and professional care by older adults. Ageing Soc 2005;25:111-30.

76 Balaban RB, Galbraith AA, Burns ME, et al. A patient navigator intervention to reduce hospital readmissions among high-risk safety-net patients: a randomized controlled trial. J Gen Intern Med 2015;30:907-15

77 Greenhalgh T, Peacock R. Effectiveness and efficiency of search methods in systematic reviews of complex evidence: audit of primary sources. BMJ 2005;331:1064-5.

78 Levac D, Colquhoun H, O'Brien KK. Scoping studies: advancing the methodology. Implementation Sci 2010;5:69.

79 Thomas J, Harden A. Methods for the thematic synthesis of qualitative research in systematic reviews. BMC Med Res Methodol 2008;8:45.

80 Pope C, Mays N, Popay J. Synthesizing qualitative and quantitative health research. UK: McGraw-Hill Education, 2007.

81 Braun V, Clarke V. Using thematic analysis in psychology. Qual Res Psychol 2006;3:77-101.

82 Moreira MA. Mapas conceituais E aprendizagem significativa San Paulo: Centauro Editora, 2010. Available: http://www.uel.br/ graduacao/odontologia/portal/pages/arquivos/NDE/MAPAS\% 20CONCEITUAIS.pdf [Accessed 29 Jun 2020].

83 Netto CM, Borém Gercina Ângela, Lima deO, et al. An application of facet analysis theory and concept maps for Faceted search in a domain ontology: preliminary studies. KO 2016;43:254-64.

84 Steele Gray C, Wodchis WP, Baker GR, et al. Mapping for conceptual clarity: exploring implementation of integrated community-based primary health care from a whole systems perspective. Int J Integr Care 2018;18:14.

85 Schwarz CM, Hoffmann M, Schwarz P, et al. A systematic literature review and narrative synthesis on the risks of medical discharge letters for patients' safety. BMC Health Serv Res 2019;19:158

86 Tahsin F, Armas A. Kirakalaprathapan a, et al. information and communication technologies (ICTs) enabling integrated primary Carefor complex patients: a protocol for a scoping review. Forthcoming.

87 The World Bank. World bank country and lending groups, 2020. Available: https://datahelpdesk.worldbank.org/knowledgebase/ articles/906519-world-bank-country-and-lending-groups [Accessed August 10 2020].

88 McGowan J, Sampson M, Salzwedel DM, et al. PRESS Peer Review of Electronic Search Strategies: 2015 Guideline Statement. J Clin Epidemiol 2016;75:40-6.

89 Lal S, Adair CE. E-mental health: a rapid review of the literature. $P S$ 2014;65:24-32

90 Bramer WM, Rethlefsen ML, Kleijnen J, et al. Optimal database combinations for literature searches in systematic reviews: a prospective exploratory study. Syst Rev 2017;6:245.

91 Tricco AC, Antony J, Zarin W, et al. A scoping review of rapid review methods. BMC Med 2015;13:224.
92 McGee S. Chapter 5 - Reliability of Physical Findings. In: McGee $\mathrm{S}$, ed. Evidence-Based physical diagnosis. Fourth Edition. Philadelphia: Content Repository Only! 2018: 27-38.

93 Waffenschmidt S, Knelangen M, Sieben W, et al. Single screening versus conventional double screening for study selection in systematic reviews: a methodological systematic review. BMC Med Res Methodol 2019;19:132.

94 Hoffmann TC, Glasziou PP, Boutron I, et al. Better reporting of interventions: template for intervention description and replication (TIDieR) checklist and guide. BMJ 2014;348:g1687.

95 Hong QN, Pluye P, Fàbregues S, et al. Improving the content validity of the mixed methods appraisal tool: a modified e-Delphi study. $J$ Clin Epidemiol 2019;111:49-59.

96 Hong QN PP, bregues S F, Bartlett G. Mixed methods appraisal too (MMAT), version 2018, 2018. Available: http://mixedmethodsappr aisaltoolpublic.pbworks.com/w/file/fetch/127916259/MMAT_2018_ criteria-manual_2018-08-01_ENG.pdf [Accessed January 292021$].$

97 Pluye P, Gagnon M-P, Griffiths F, et al. A scoring system for appraising mixed methods research, and concomitantly appraising qualitative, quantitative and mixed methods primary studies in mixed studies reviews. Int J Nurs Stud 2009;46:529-46.

98 Pace R, Pluye P, Bartlett G, et al. Testing the reliability and efficiency of the pilot mixed methods appraisal tool (MMAT) for systematic mixed studies review. Int J Nurs Stud 2012;49:47-53.

99 Wilson AN, Spotswood N, Hayman GS. Improving the quality of maternal and newborn care in the Pacific region: a scoping review. Lancet Reg Health - Western Pacific 2020;3.

100 El-Awaisi A, Joseph S, El Hajj MS, et al. A comprehensive systematic review of pharmacy perspectives on interprofessional education and collaborative practice. Res Social Adm Pharm 2018;14:863-82.

101 Chu $\mathrm{CH}$, Ploeg J, Wong R, et al. An integrative review of the structures and processes related to nurse Supervisory performance in long-term care. Worldviews on Evidence-Based Nursing 2016;13:411-9.

102 Scott SD, Rotter T, Flynn R, et al. Systematic review of the use of process evaluations in knowledge translation research. Syst Rev 2019;8:266

103 Care CEPaOo. Describing interventions in EPOC reviews, 2017. Available: https://epoc.cochrane.org/sites/epoc.cochrane.org/ files/public/uploads/Resources-for-authors2017/describing interventions in epoc reviews.pdf [Accessed June 3 2020].

104 Arksey H, O'Malley L. Scoping studies: towards a methodological framework. Int J Soc Res Methodol 2005;8:19-32.

105 Shamseer L, Moher D, Clarke M, et al. Preferred reporting items for systematic review and meta-analysis protocols (PRISMA-P) 2015: elaboration and explanation. BMJ 2015;349:g7647.

106 Golinelli D, Boetto E, Carullo G, et al. Adoption of digital technologies in health care during the COVID-19 pandemic: systematic review of early scientific literature. J Med Internet Res 2020;22:e22280.

107 Adjekum A, Blasimme A, Vayena E. Elements of trust in digital health systems: Scoping review. J Med Internet Res 2018;20:e11254

108 Punch KF. Developing effective research proposals. 2nd ed. Thousand Oaks, CA: Sage, 2006.

109 Øyeflaten I, Johansen T, Nielsen CV. eHealth interventions to facilitate work participation: a scoping review protocol. JBI Evid Synth 2019;17

110 Lockwood C, dos Santos KB, Pap R. Practical guidance for knowledge synthesis: Scoping review methods. Asian Nurs Res 2019;13:287-94.

111 Lewis CC, Boyd MR, Walsh-Bailey C, et al. A systematic review of empirical studies examining mechanisms of implementation in health. Implementation Sci 2020;15:21. 